"character" and eventually render them "expendable" (pp.21 f). A series of arms control measures is seen fit to bring these changes about. To preserve and consolidate whatever progress is being achieved in the process, Bahr relies on international legal provisions.

Common security is clearly tailored for Europe. Though, in principle, Bahr's concept is also applicable to third world conflicts, here its prerequisite seems to be absent. It is questionable whether the threat of nuclear war is felt strongly and widely enough in the North, in order to enforce action of the kind Bahr suggests. But less likely still, fear of mutual nuclear extermination will possibly take precedence over less arcane and far more pressing problems in the South, such as famine, utter poverty, civil war, and any one of those various choices from the great powers' interventionist repertoire. Not surprisingly, then, common security has encountered little enthusiasm, reservations, and criticism on part of third world scholars. However, none of this is reflected in the book. The last essay, intended to describe how the concept was received outside Germany and commissioned to a junior member of the IFSH-staff, does not mention these voices at all.

Whatever its merits, shortcomings, or limitations: common security will certainly be part of the domestic German debate on peace and security for some time to come. The present book offers the opportunity to get acquainted with an alternative security concept likely to gain wider acceptance than its less prominently patronized equals. Those readers, however, who are interested in the concept's implications for the so-called third world, should either rest fairly content with this review or consult the bibliography appended to the book. And those, who would like to know more about the part international law is supposed to play with respect to common security, will have to await future elaborations by the concept's advocates. On this particular aspect the book offers close to nothing.

Thomas Horlohe

\title{
Die Europäische Gemeinschaft in der Weltwirtschaft
}

Dokumente zum 6. Malenter Symposion

Edition Dräger-Stiftung, herausgegeben von Lothar Späth und Christian Dräger Nomos Verlagsgesellschaft, Baden-Baden 1987, 410 Seiten, DM 45,-

Seit der Süderweiterung der Europäischen Gemeinschaft am 1. 1. 1986 kann deren Bedeutung für die Weltwirtschaft kaum mehr bezweifelt werden. Mit einem Anteil von über $60 \%$ am Bruttosozialprodukt der westlichen Wirtschaft und 320 Millionen potentiellen Verbrauchern in einem einheitlichen Binnenmarkt ohne Grenzen, dessen Vollendung bis 1992 angestrebt wird, spielt die EG neben den USA und Japan eine entscheidende Rolle bei der Ausgestaltung der Weltwirtschaft sowie der internationalen Handelsbeziehungen. 
Angesichts der herausragenden Stellung der EG kann es dann auch kaum verwundern, daß namhafte Persönlichkeiten aus Wissenschaft, Wirtschaft, Politik und Verwaltung im Rahmen des 6. Malenter Symposions diesen Themenkomplex zum Gegenstand ihrer Tagung gemacht haben. Wie sich schon dem Titel des Tagungsberichts entnehmen läßt, kann sich der einschlägig interessierte Leser anhand der im vorliegenden Buch zusammengefaßten Referate sowie Berichte der verschiedenen Arbeitskreise eingehend über "Die Europäische Gemeinschaft in der Weltwirtschaft " informieren. Dabei kommen sowohl ihre Beziehungen zu anderen Industrienationen als auch diejenigen zu Ländern der sogenannten Dritten Welt sowie die Notwendigkeit eines freien Welthandels zur Sprache. Die Vielzahl der beteiligten Autoren gewährleistet zugleich einen möglichst umfassenden Einblick in die verschiedensten Aspekte des behandelten Themenkreises.

An der Spitze der "einführenden Referate" steht ein Vortrag von Karl-Heinz Narjes über "Die Europäische Gemeinschaft am Scheideweg zwischen Integration und Renationalisierung ". Die steigende Abhängigkeit weltwirtschaftlicher und -politischer Entscheidungen erfordert demnach ein handlungs- und entscheidungsfähiges Europa. Voraussetzung dafür sind Reform und Revitalisierung der EG, wobei insbesondere die Schaffung eines einheitlichen Binnenmarktes bis 1992, eine kooperative Wachstumstrategie sowie technologische und institutionelle Neuerungen von Bedeutung sind. Die Einigung Europas ist jedoch wegen des immer wieder erforderlichen Verzichts auf nationale mitgliedstaatliche Kompetenzen nur schrittweise zu erreichen.

Wenn die Ausführungen des Referats bis hierhin auch als allgemein bekannt vorausgesetzt werden dürften, so folgt anschließend eine interessante These: "Integration und Renationalisierung schließen einander nicht von vornherein zwingend aus" (Seite 27). Wie und ob allerdings eine zusätzliche Wahrnehmung von Aufgaben durch die Europäische Gemeinschaft bei gleichzeitiger Rückübertragung von Kompetenzen auf die Mitgliedstaaten funktioniert, darauf geht der Verfasser leider in seinen weiteren Ausführungen nicht mehr ein.

Der Vortrag "Eurosklerose, Europhorie oder Revitalisierung der europäischen Wirtschaft?" von Herbert Giersch beschäftigt sich im Anschluß an eine Definition des Begriffs "Eurosklerose" als Inflexibilität der Märkte mit einem Vergleich der Beschäftigungs- und Arbeitslosenquoten in Europa, Amerika und Japan als deutlichstem unter anderen Indikatoren für besagte Inflexibilität des europäischen Marktes. Der Verfasser wirft Europas Märkten in der Hauptsache ein Mangel an Offenheit vor und plädiert darum für ein "radikales" Programm der Offenheit, das gerichtet ist auf die Durchsetzung der Marktwirtschaft im nationalen, europäischen und internationalen Handel. Schlagwörter sind in diesem Zusammenhang Dezentralisierung, Privatisierung, Entregulierung als Bedingung für die Schaffung des gemeinsamen Binnenmarktes bis 1992, Liberalisierung und Steuersenkung. In erster Linie sollen die Konsumenten die Regelungen bestimmen, die den Gemeinsamen Markt gestalten, während die behördliche Intervention auf Mindestregeln für den Gesundheits- und Umweltschutz beschränkt bleibt.

Schließlich wird darauf hingewiesen, daß nicht nur die Liberalisierung innerhalb der EG, 
also die Schaffung des gemeinsamen Binnenmarktes bis 1992, sondern vielmehr die Außenliberalisierung von hervorragender ökonomischer Bedeutung ist, an deren Ziel ein freier Welthandel zugunsten aller Beteiligten steht. Daß allerdings die vollständige Außenliberalisierung des Gemeinsamen Marktes ohne die Gewährleistung eines vergleichbaren Verhaltens auf Seiten so wichtiger Handelspartner wie der USA und Japan tatsächlich die gewünschten Auswirkungen mit sich bringt, kann mit guten Gründen auch in Frage gestellt werden.

Karl-Heinz Wessel geht in seinem Referat "Die Rolle der Europäischen Gemeinschaft auf den internationalen Finanzmärkten " zunächst der Frage nach, ob es überhaupt eine europäische Finanzgemeinschaft gibt. Trotz beachtlicher Fortschritte auf dem Gebiet der Währungspolitik, der im EWG-Vertrag nur eine untergeordnete Bedeutung zukommt, sowie zunehmender Aktivitäten europäischer Finanzinstitutionen, wie etwa der Europäischen Investitionsbank, spricht die bisherige Entwicklung auf dem Kapitalmarkt entscheidend gegen das tatsächliche Bestehen einer solchen Gemeinschaft. Denn von einer wirklichen Liberalisierung des Kapitalverkehrs innerhalb der EG kann in vielen Bereichen kaum die Rede sein.

Im folgenden werden die Beziehungen zwischen der EG und den internationalen Finanzmärkten beleuchtet. Anhand von Zahlen und Statistiken kann dabei nachgewiesen werden, daß die Europäischen Gemeinschaften erheblich von den internationalen Finanzmärkten, diese aber umgekehrt auch vom Bestehen und Agieren der Europäischen Gemeinschaften profitiert haben.

Die beiden letzten "einführenden Referate" befassen sich mit den Problemen und Chancen, die die Süderweiterung der EG mit sich gebracht hat. Dabei geht Hans-Jürgen Krupp auf die in diesem Zusammenhang vorliegenden wirtschaftlichen Aspekte ein, wie sie sich aus der Sicht der ursprünglichen Mitgliedstaaten darstellen. Probleme bereitet die europäische Agrarpolitik, die sich durch die Süderweiterung und der damit verbundenen Zunahme des landwirtschaftlichen Produktivitätspotentials neuen Aufgaben und Anforderungen gegenübergestellt sieht. Zugleich bedingen der Beitritt Griechenlands, Spaniens und Portugals eine Einbeziehung der Nord-Süd-Problematik. Die Voraussetzungen für einen Binnenmarkt 1992, der das Adjektiv "einheitlich" verdient, müssen in den verbleibenden Jahren durch eine verstärkte Entwicklungspolitik erst einmal geschaffen werden. Ob angesichts der kumuliert auftretenden Schwierigkeiten die Süderweiterung der EG eine realistische Chance für einen Neuansatz zur Schaffung einer "echten Wirtschaftsgemeinschaft" bietet, wie sie vom Verfasser gesehen wird, werden erst die nächsten Jahre zeigen können.

Eduardo de Sousa Ferreira behandelt in seinem Referat dieselbe Thematik aus der Sicht Portugals und stellt dabei in erster Linie die Probleme dar, die die Integration in die EG innerhalb Portugals hervorruft.

Nach diesen "einführenden Referaten" wird in einem umfassenden zweiten Teil des vorliegenden Buches über die Ergebnisse der einzelnen Arbeitskreise des Symposions be- 
richtet. Dabei werden die im ersten Teil angesprochenen Aspekte aufgegriffen und eingehend diskutiert.

Zusammenfassend bleibt festzustellen, daß das besprochene Buch durchaus dazu geeignet ist, den Leser in die Rolle der Europäischen Gemeinschaft in der Weltwirtschaft einzuführen. Obgleich schon im Hinblick auf den behandelten Themenkreis der Tagung rechtliche Fragen naturgemäß nicht im Vordergrund standen, kann sich doch auch der gemeinschaftsrechtlich interessierte Jurist mit der Lektüre dieses Buches in einigen Bereichen brauchbare und nützliche (Hintergrund-)Informationen verschaffen.

Roger Kampf

\section{Peter Hammans}

\section{Das politische Denken der neueren Staatslehre in der Bundesrepublik}

Opladen, Westdeutscher Verlag, 1987, VIII/334 S., DM 49,-

Rechtswissenschaft ist die Wissenschaft vom geltenden Recht. Sie hat die vorhandene Rechtsordnung zum Gegenstand. In diesem Kontext beschäftigt sie sich auch mit den Entstehungs- und Anwendungsbedingungen von Rechtsnormen. Weil sie dies tut, richtet sich ihr Augenmerk auf den Staat. Dieser ist die maßgebliche Instanz der Rechtssetzung und -vollziehung. Und weil er dies ist, ist er auch Endpunkt politischer Interessenartikulation aus der Umwelt und zugleich Ausgangspunkt politischer Interessenvermittlung, die sich in der Rechtsordnung äußert. Ist so der Staat Medium der Transformation politischer Belange in objektives Recht, so gehören Staat und Recht notwendig zusammen. Sicherlich ist dies nur ein Teilaspekt staatlichen Wirkens; aber es ist eben dieser Teilaspekt, welcher den Staat zum Gegenstand der rechtswissenschaftlichen Staatslehre macht. Damit überschneidet sich diese Disziplin hinsichtlich ihres Gegenstandes partiell mit demjenigen der Politikwissenschaft. Eine derartige Duplizität wissenschaftlicher Befassung mit einem einzelnen Gegenstand ist nichts Ungewöhnliches; im Gegenteil. So kann beispielsweise ein einziges Mineral Gegenstand der Geologie, der Chemie, der Physik und der Paläontologie sein, ohne daß solche Vielfalt als problematisch empfunden würde. Das gilt in ähnlicher Weise für den Staat, der nahezu in allen sozial- und den meisten geisteswissenschaftlichen Disziplinen "vorkommt". Günstigstenfalls kann unter solchen Bedingungen die eine von der anderen Disziplin profitieren. Schon unter diesem Aspekt ist eine politikwissenschaftliche Analyse der Staatslehre zu begrüßen.

Das Ergebnis der vorliegenden Untersuchung läßt sich in einem einzigen Satz zusammenfassen: Die juristische Staatslehre verharrt in einem politischen Konservatismus. Wie kommt eine solche Diagnose, die immerhin u.a. das Schaffen von E. W. Böckenförde, F. Ermacora, E. Fraenkel, E. Forsthoff, R. Herzog, K. Hesse, M. Kriele, H. Krüger, 